\title{
August 2018 Critical Care Case of the Month
}

\author{
Emma Simpson, MD \\ Banner University Medical Center Phoenix \\ Phoenix, AZ USA
}

\section{History of Present IIIness}

A 19-year-old gravida 1, para 0 woman in her early second trimester presented to the Emergency Department with intractable vomiting, green sputum icteric sclerae, chest pain, palpitations and weakness for one week prior to presentation. She was visiting the US from an island in Micronesia. The patient has been experiencing feelings of general malaise since the beginning of her pregnancy: she experienced severe nausea and vomiting throughout her first trimester, and a $4.5 \mathrm{~kg}$ weight loss in the 2 months prior to presentation.

\section{$\mathrm{PMH}, \mathrm{SH}, \mathrm{FH}$}

Before becoming pregnant, the patient was active and healthy. She does not smoke and her family history is unremarkable.

\section{Physical Examination}

Physical exam showed a thin, small young woman. Her physical examination showed a tachycardia of 114 and icteric sclera but was otherwise unremarkable.

Which of the following should be done?

1. Admit to the hospital with measurement of electrolytes, transaminases and bilirubin

2. Discharge to home with a prescription for pyridoxine/doxylamine

3. Ultrasound

4. 1 and 3

5. All of the above 


\section{Correct!}

\section{1 and 3}

The patient has hyperemesis gravidarum. Although $50 \%$ of women have vomiting during the first trimester of pregnancy and additional $25 \%$ have nausea, hyperemesis gravidarum has been defined as more than three episodes of vomiting per day such that weight loss of $5 \%$ or three kilograms has occurred and ketones are present in the urine (1). Many consider this a medical emergency and the patient should be admitted to the hospital for observation and treatment.

Our patient was mildly hypokalemic and hyponatremic with mildly elevated transaminases and bilirubin.

Which of the following are possible causes of hyperemesis gravidarum?

1. Choriocarcinoma

2. Hepatitis

3. Urinary tract infection

4. 1 and 3

5. All of the above 


\section{Correct! \\ 5. All of the above}

There a number of causes of hyperemesis gravidarum (1). These include infections (urinary tract infection, hepatitis, meningitis), gastrointestinal disorders $(H$. pylori, gastroenteritis, appendicitis, cholecystitis, pancreatitis, small bowel obstruction), metabolic disorders (thyrotoxicosis, Addison's disease, hyperparathyroidism), drugs (antibiotics, iron supplements), gestational trophoblastic diseases, molar pregnancy, and choriocarcinoma.

What additional laboratory should be drawn?

1. Free $\mathrm{T} 4$

2. Human chorionic gonadotrophin (hCG)

3. Urinalysis

4. 1 and 3

5. All of the above 


\section{Correct! \\ 5. All of the above}

Urinalysis showed and elevated and the presence of ketones but was otherwise unremarkable. Human chorionic gonadotrophin (hCG) was 319,864 (normal: 13,300254,000 mIU/L) and free T4 >8 (normal: 0.78-1.33 nG/dL).

Which of the following are true regarding the elevated hCG and T4?

1. Elevated hCG is anticipated in the first trimester

2. Elevated hCG is diagnostic of choriocarcinoma

3. Elevated $\mathrm{T} 4$ is anticipated in the first trimester

4. 1 and 3

5. All of the above 


\section{Correct! \\ 1. Elevated hCG is anticipated in the first trimester}

hCG is produced by the placenta and elevated during the first trimester of pregnancy and declines over the next two trimesters. The most commonly accepted theory of hyperemesis gravidarum suggests that these high levels of $h C G$ account for the nausea and vomiting and the decline in these complaints in the second and third trimester (1). hCG is often markedly elevated with choriocarcinoma although it can be only mildly elevated. Free T4 does increase in pregnancy by up to $50 \%$ (2). However, the T4 is elevated to a much higher level than expected. Our patient's thyroid stimulating hormone (TSH) was $<0.01$ (normal: $0.2-3.0 \mathrm{mIU} / \mathrm{L}$ ). Thyroid ultrasound imaging showed no nodules or goiter. Thyroid stimulating immunoglobulins (TSI) was $<89 \%$ of baseline (normal: $<140 \%$ ). A Burch-Wartofsky score of $>45$ was calculated and a presumptive diagnosis of gestational transient thyrotoxicosis was made (3). Our patient was admitted to the intensive care unit, treated with IV fluids and electrolytes and consultation by hepatology and endocrine.

What additional therapy should be given for her thyrotoxicosis?
1. Beta blockers
2. $\left.\right|^{131}$ for thyroid ablation
3. Propylthiouracil (PTU)
4. 1 and 3
5. All of the above 


\section{Correct!}

\section{1 and 3}

Treatment of gestational transient thyrotoxicosis is similar to thyrotoxicosis in nonpregnant patients except that radioactive iodine should never be given to a pregnant patient since it crosses the placenta and will also ablate the fetus' thyroid $(4,5)$. Propylthiouracil (PTU) or methimazole should be given to decrease synthesis of T4, beta blockers to control sympathetic overload and dexamethasone to decrease peripheral conversion from T4 to T3.

Gestational transient thyrotoxicosis is caused by stimulatory action of hCG on the thyroid receptor $(4,5)$. It is clinically similar to Graves' disease although is typically less severe in pregnant women than Graves' and is considered less harmful to mother and fetus than an autoimmune cause of hyperthyroidism. The two disorders are distinguished by the presence or absence of thyroid stimulating immunoglobulin.

Our patient was evaluated by hepatology who thought her elevated transaminases and bilirubin were likely the result of her thyrotoxicosis. She was treated with PTU, propranolol and dexamethasone. Her free T4 gradually trended down over the course of 4 days and she was transitioned to the antepartum floor. PTU was stopped on the day of discharge. The patient will be followed as an outpatient for tapering of her propranolol and repeat TSI. She will also follow up in high-risk pregnancy clinic.

\section{References}

1. Jueckstock JK, Kaestner R, Mylonas I. Managing hyperemesis gravidarum: a multimodal challenge. BMC Med. 2010 Jul 15;8:46. [CrossRef] [PubMed]

2. Fryhofer SA. Guidelines for thyroid disease in pregnancy: Key points. Medscape. November 09, 2011. Available at: https://www.medscape.com/viewarticle/752878 (accessed 7/24/18).

3. MDCalc. Burch-Wartofsky point scale (bwps) for thyrotoxicosis. Available at: https://www.mdcalc.com/burch-wartofsky-point-scale-bwps-thyrotoxicosis (accessed 7/24/18).

4. Bernstein PS, Koscica KL. Thyrotoxicosis in pregnancy. Medscape. April 15, 2003. Available at: https://www.medscape.com/viewarticle/451718\#vp 1 (accessed 7/24/18).

5. Cooper DS, Laurberg P. Hyperthyroidism in pregnancy. Lancet Diabetes Endocrinol. 2013 Nov;1(3):238-49. [CrossRef] [PubMed] 